\title{
On-surface synthesis of two-dimensional polymers: Rational design and electronic properties
}

\author{
Sabine Maier \\ Department of Physics, Friedrich-Alexander University Erlangen-Nürnberg, Erwin-Rommel- \\ Strasse 1, 91058 Erlangen, Germany. \\ e-mail: sabine.maier@fau.de
}

\begin{abstract}
The fabrication of long-range ordered 2D polymers directly on metal surfaces still presents a great challenge in ultra-high vacuum surface science. The structure of the polymer networks is in general predetermined by the coupling chemistry and the symmetry of the molecular precursors. However, the irreversible nature of the $\mathrm{C}-\mathrm{C}$ coupling reaction readily leads to the formation of defects. Over the last decade, several strategies in the on-surface synthesis have been suggested to improve the structural order. Among them, the programmed hierarchical synthesis through a sequential polymerization proved to be suitable to reduce defects in the formation of $2 \mathrm{D}$ polymers. This chapter provides a review on the state-of-the art structural characterization of surface-supported 2D polymers by established surface science techniques. Further, the chapter focuses on the electronic structure of 2D polymers, which remained experimentally widely unexplored until now.
\end{abstract}

\section{Introduction}

Two-dimensional polymers are sheet-like, covalently bonded molecular networks that extend in exactly two dimensions. ${ }^{1-2}$ The ultimate goal in fabricating novel 2D polymeric materials is motivated by a potentially limitless range of structures with unprecedented properties that can be created. In these organic analogues of graphene, the band gap and the charge carrier mobility may be tuned by using specifically designed molecular building blocks. On-surface polymerization proved successful for the fabrication of one-dimensional structures with atomically defined width, edge termination, and dopants on the surface. ${ }^{3-11}$ In contrast, the bottom-up fabrication of crystalline 2D polymers presents a far greater challenge owing to the limited control during their synthesis on the surface despite the wide range of possible surface reactions. ${ }^{12}$ Some of the main issues, are the bond flexibility in the molecular building blocks that allows the formation of a range of polygons and the irreversible nature of the created covalent $\mathrm{C}-\mathrm{C}$ bonds that prohibits an error correction during the network formation in ultra-high vacuum (UHV). These issues lead readily to equilibrium disordered structures of limited size. ${ }^{13}$ Hence, the on-surface 
synthesis of two-dimensional periodic structures and the characterization of their structural and physical properties is a growing field of research.

The 2D covalent organic polymers hold great potential in particular for applications in future electronic and optoelectronic devices. The strong coupling between the molecular building blocks by covalent bonds promotes, apart from a great mechanical and thermal stability, the ability to conduct electrons in the extended $\pi$-conjugated system. The inherent porous structure induces an electronic band gap that can be controlled by the pore size, density, and geometry. ${ }^{14}$ The topology of the network may lead in some cases to unique electronic band structures with linear electronic band dispersion and associated Dirac physics as predicted by density-functional theory (DFT).${ }^{15-17}$ Hence, some of the 2D polymers promise to combine the outstanding electronic properties of graphene with a tunable band gap, which makes them more suitable than pristine graphene for many electronic and optoelectronic applications that require gapped semiconductor materials. ${ }^{4}$ In addition, the use of functionalized building blocks allows for chemically tuning the electronic properties through doping. However, requirement for these exciting electronic properties are long-range ordered structures. The electronic structure of bottom-up fabricated 2D networks remained experimentally so far widely unexplored due to a lack of structural order. Moreover, the pores of the 2D polymers offer fascinating options in host-guest chemistry. Hence, 2D polymer networks may be suitable for future applications in molecular electronics and as membranes for gas sensing, separation, and storage owing to their tunable porous structure and their improved thermal stability compared to non-covalently linked self-assemblies. ${ }^{2,18-19}$

Despite the numerous potential applications, the synthesis of large-scale, periodic 2D polymers via wet chemistry or by on-surface synthesis is still challenging. In organic chemistry, mainly boronic acid-based condensation and Schiff-base reactions have been applied as major reversible reactions so far to successfully fabricate covalent organic frameworks. ${ }^{20}$ Numerous graphene-like, 2D all-carbon materials have been successfully synthesized in flask. ${ }^{21}$ However, the synthesis of a whole range of novel 2D carbon allotropes such as graphyne and graphdiyne could so far not be accomplished. ${ }^{22}$ In this respect, on-surface synthesis may be a powerful method, since it has been shown that atomically defined carbon-based nanostructures can be fabricated in a bottom-up approach directly on the surface. ${ }^{23-26}$ Apart from acting as catalyst, the surface provides a templating effect for the fabrication of novel 2D materials by confining the reacting species in two dimensions. Thereby, the molecular precursors are commonly activated by thermal annealing, but also irradiation with electrons or light ${ }^{27-28}$ have been successfully applied. For their characterization, various surface science techniques are used. However, scanning probe microscopy (SPM) is currently one of the favorite characterization tools for the surface supported 2D polymers, since molecular resolution is readily accomplished and imaging in real space facilitates a direct assessment of the structural quality. While scanning tunneling microscopy (STM) and spectroscopy provide direct insights into the local topographic and electronic structure, non-contact atomic force (nc-AFM) microscopy has proven important in the unambiguous identification of reaction 
mechanisms and intermediates, since functionalized tips allow to resolve the molecular structure with submolecular resolution..$^{29-30}$ Complementary, X-ray photoelectron spectroscopy (XPS) is commonly used to determine the chemical state of the reaction products, near edge X-ray absorption fine structure (NEXAFS) to obtain information on the adsorption geometry, and angle-resolved photoemission spectroscopy (ARPES) to determine the band dispersion.

In this chapter, recent progress towards the synthesis of ordered 2D surface-supported polymers is reviewed. Thereby, their geometric structure as consequence of various synthesis strategies as well as their electronic structure are discussed.

\section{Bottom-up fabrication of $2 D$ networks}

\subsection{Limited structural control}

One of the key issues preventing structural control in the bottom-up fabrication of periodic 2D polymers under UHV condition is the irreversible nature of the newly formed intermolecular covalent bonds. In contrast to self-assembled supramolecular structures, which are held together by weak, reversible interactions - i.e. hydrogen bonds, van-der-Waals, or metal-ligand interactions - the covalent bond prohibits an error correction in the network formation. Therefore, the experimentally observed 2D network are usually kinetically trapped far from equilibrium. Coupling reactions used in dynamic covalent chemistry, a common concept in supramolecular chemistry that is based on the reversible bond formation, were so far only applied with limited success in on-surface polymerization experiments in UHV. One of the reasons is the irreversible loss of smaller products, e.g. water, which are essential to realize the back-reaction. For instance, Abel and co-workers ${ }^{31}$ investigated aryl boronic acids as building blocks, but could not achieve periodic networks on a $\mathrm{Ag}(111)$ surface as a consequence of the irreversible bond formation in UHV. In contrast, Yaghi et al. ${ }^{32}$ employed successfully the same coupling reaction in a solution approach to obtain the first covalent organic frameworks (COF). Similarly, branched and irregular network structures formed by condensation polymerization of trialdehydes and diamines co-adsorbed on $\mathrm{Au}(111)$ in UHV, which is a reversible reaction in solution. ${ }^{33}$

As a consequence of the irreversible bond formation, polygonal defects are frequently observed in 2D networks fabricated in UHV. The defect density is strongly related to the bond flexibility in the molecular building blocks. The energy relaxation for arranging threefold symmetric molecules into isolated, regular $n$-gons favors the hexagon, whose geometry is commensurate with the symmetry of the molecule. ${ }^{13,}{ }^{34}$ However, molecules may form other polygons, at an energy cost of around an $\mathrm{eV}$ per ring, which leads readily to equilibrium disordered structures. In 
conclusion, 2D covalent networks are inherently limited with respect to their structural perfection. ${ }^{13}$ The structural quality of covalent networks can however be improved by an optimization of the reaction parameters, as it was shown in the kinetically controlled Ullmann-type coupling on $\mathrm{Au}(111) .{ }^{35}$

In addition, we have also to consider effects of the molecule-surface interaction on the structural control in the bottom-up fabrication of $2 \mathrm{D}$ networks. The substrate does not only act as a support but is actively involved in all reaction steps and therefore significantly influences the morphology of the self-organized covalent nanostructures. Bieri et al. showed in an Ullmann-type coupling reaction that polyphenylene networks adapt morphologies from branched, fractal-like structures on $\mathrm{Cu}(111)$ to extended, regular 2D networks on $\mathrm{Ag}(111) .{ }^{36}$ Their DFT analysis of the diffusion and coupling pathways on $\mathrm{Cu}$ and $\mathrm{Ag}$ revealed that the balance between the diffusion and coupling steps is significantly different on the two substrates and plays an important role in the structural control. While the radicals after debromination spontaneously form covalent intermolecular bonds on $\mathrm{Cu}$, on the $\mathrm{Ag}$ surface, diffusion clearly prevails over intermolecular coupling, which results in an overall increased mobility of the molecules on the surface and in the formation of regular 2D networks. Hence, a high mobility (or low coupling affinity of the reactants) is a prerequisite for the growth of dense $2 \mathrm{D}$ polymer networks. In addition, also adsorbed side products from the on-surfaces synthesis can influence the diffusion of the networks and prohibit the fabrication of extended two-dimensional networks.

\subsection{Rational design of $2 D$ polymers}

A crucial step in the rational design of $2 \mathrm{D}$ polymers in on-surface synthesis experiments is to identify reaction protocols that irrespective of the reaction kinetics lead to periodic networks. The molecular design of the building blocks is hereby a crucial step to obtain 2D polymers with a well-defined structure. Key parameters for the structural variety and control are their symmetry, rigidity of the molecular scaffold, and functional groups.

In general, the networks symmetry affects the susceptibility to form defects; the angular strain to form irregular polygons increases by about a factor two for fourfold-symmetric networks compared to threefold-symmetric networks. Therefore, the likeliness for the irregular polygon formation is drastically reduced in a squared network, while nearly inevitable for honeycomb networks built from larger compounds without using a rational design. The geometrical stability of two-fold symmetric networks was already demonstrated in the pioneering work by Grill et al. in the first thermally induced on-surface Ullmann-type coupling. They could obtain defect-free patches of covalently-linked tetra(4-bromophenyl)porphyrins. ${ }^{37}$ For honeycomb networks, a rigid molecular scaffold of the precursors is crucial to improve the structural quality, because weak and rigid bonds yield a highly ordered network, while strong and flexible bonds give rise to disordered structures. ${ }^{13}$ The prevalence of hexagonal pores has been successfully shown for example for the 
rigid hexaiodo-substituted macrocycle cyclohexa-m-phenylene precursor, that has been applied to synthesize porous graphene (see Fig. 1a). ${ }^{38}$

Conformationally rigid precursors
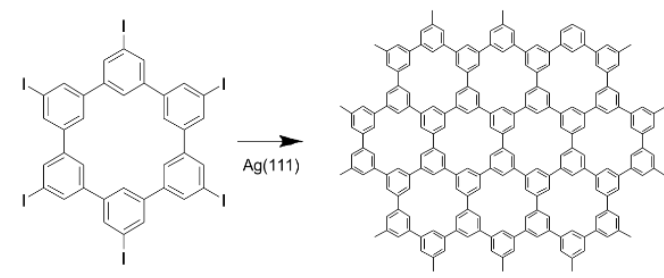

Cyclotrimerization reactions

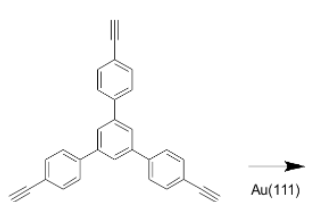

$$
\text { . }
$$

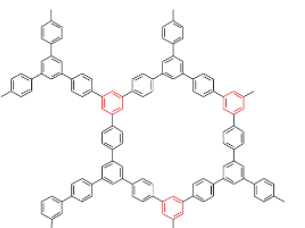
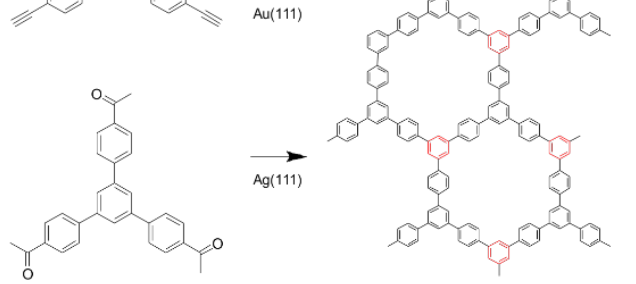
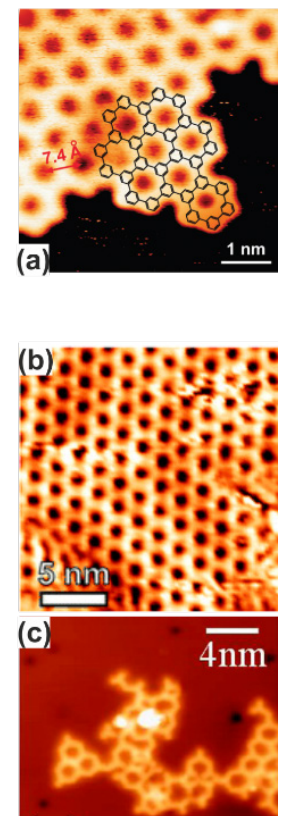

Fig. 1. Selected design strategies in the on-surface synthesis of ordered 2D polymers. (a) Formation of porous graphene via an Ullmann-type reaction of the hexaiodo-substituted macrocycle cyclohexa-m-phenylene on $\mathrm{Ag}(111)$. Adapted with permission from Ref. ${ }^{38}$, copyright 2015, RCS. (b)-(c) Cyclotrimerization of (b) 1,3,5-tris(4-ethynylphenyl)benzene and (c) 1,3,5-tris(4acetylphenyl)benzene into 2D conjugated covalent networks. (b) Adapted with permission from Ref. ${ }^{39}$,copyright 2014, RCS and (c) adapted with permission from Ref. ${ }^{40}$, copyright 2015 , ACS.

Ullmann-type coupling is the best studied and most applied polymerization reaction on surfaces. However, organic chemistry is extremely diverse; thus, a good strategy is to explore different coupling reactions on surfaces that provide more geometrical stability. In contrast to linear reaction schemes, cycloadditions and cyclization reactions have great potential for the fabrication of defect-free 2D polymers in onsurface chemistry, since byproducts in this "node design" are rarely generated. The high selectivity of the cyclotrimerization reaction was convincingly demonstrated in the on-surface synthesis of well-ordered, two-dimensional networks on Au(111) using 1,3,5-tris(4-ethynylphenyl)benzene (TEB) by Liu et al. (see Fig. 1b), while homocoupling of the same precursor on $\mathrm{Ag}(111)$ led to unordered networks. ${ }^{41}$ Also the cyclotrimerization of acetyls to aromatics has shown to be a promising approach to form $2 \mathrm{D}$ conjugated covalent networks on surfaces under UHV (see Fig. 1c). ${ }^{40}$ 
The underlying substrate provides also for templating effects to promote long-range order. For instance, intrinsic adatoms form intermediate organometallic structures with carbon-metal-carbon bonds in the Ullmann-type reaction on the $\mathrm{Cu}$ and $\mathrm{Ag}$ surfaces instead of directly establishing covalent bonds. These organometallic structures can serve as a template considering the advantage of the reversibility of organometallic networks to equilibrate into highly ordered structures in a self-assembly process. However, the covalent-linking is far from being a topochemical reaction, because the conversion from organometallic to covalent structures is accompanied by a considerable shrinkage of intermolecular distances, which makes it difficult to obtain very large domains. ${ }^{42}$ In topochemical polymerization reactions the monomers polymerize without the movement of their center of gravity within the molecular lattice, only a minimal movement around the polymerizable group is allowed while the rest remains static. Diacetylene compounds are popular to proceed in topochemical polymerizations. ${ }^{43}$

\subsection{Hierarchical structure formation}

Exploring strategies for including hierarchical structure formation is a major step towards increasing the complexity in on-surface synthesis experiments. In a hierarchical synthesis, sequential polymerization is applied using the same or dissimilar reaction types to improve the structural quality of the reaction products. An advantage of the sequential polymerization is the fabrication of geometrically stable and larger intermediate structures, which would be too large for thermal deposition. Sequential on-surface synthesis strategies comprising two different reaction types are commonly applied in one-dimensional structures. The fabrication of graphene nanoribbons often combines an Ullmann-type coupling reaction with a subsequent intramolecular Scholl reaction (cyclodehydrogenation) to planarize the structures. ${ }^{3-}$

4, 44 Also intermolecular dehydrogenation reactions combined with Ullmann-type coupling are used in 1D structures, for instance, to increase the width of graphene nanoribbons. ${ }^{9,}, 45$

In the hierarchical synthesis of two-dimensional networks mainly the sequential synthesis via the same reaction type has been employed. In Ullmann-type coupling reactions, one takes the advantage of the bond energy differences between C-I, C$\mathrm{Br}$, and $\mathrm{C}-\mathrm{Cl}$ bonds, which leads to a lower activation temperature for aryl iodides than aryl bromides and aryl chlorides. On $\mathrm{Au}(111)$, the reaction hierarchy is preserved because stable organo-metallic intermediates are only rarely observed, ${ }^{46}$ which makes it an ideal substrate. In the pioneering work done by Lafferentz et al. ${ }^{47}$, the successful interlinking of porphyrin building blocks was demonstrated in a hierarchical manner through Ullmann-type coupling on $\mathrm{Au}(111)$ (see Fig. 2a). Thereby, selective activation of the weaker C-I bonds was used to form 1D polymer chains in a first step, which were laterally interconnected by subsequent thermal activation of $\mathrm{C}-\mathrm{Br}$ bonds at higher temperatures to obtain $2 \mathrm{D}$ network structures with high regularity. 
For the hierarchical fabrication of open-porous honeycomb networks, Eichhorn et al. used 1,3-bis(-bromophenyl)-5-(p-iodophenyl) benzene to create in a first step dimers via $\mathrm{C}$-I cleavage and subsequently $2 \mathrm{D}$ networks via $\mathrm{C}-\mathrm{Br}$ cleavage (see Fig. 2b). ${ }^{35}$ In a similar way, Shi et al. used 4-bromo-4" '-chloro-5' -(4-chlorophenyl)$1,1^{\prime}: 3^{\prime}, 1^{\prime \prime}$-terphenyl to form dimers in a $\mathrm{C}-\mathrm{Br}$ cleavage reaction and subsequently networks in a $\mathrm{C}-\mathrm{Cl}$ cleavage reaction. However, in both studies no substantial differences could be found in the network quality for the direct and hierarchical polymerization protocols. This suggests that dimers as intermediate products due their large structural flexibility do not provide a suitable template to prevent the formation of pentagonal and heptagonal pores in hexagonal networks. ${ }^{48}$

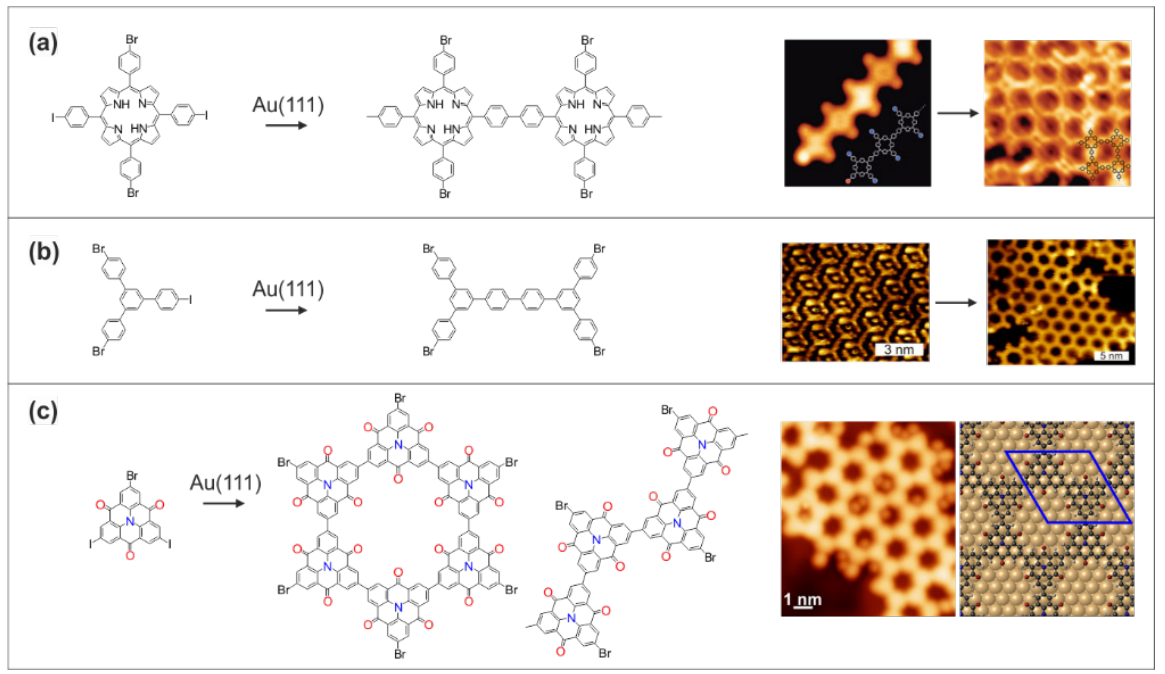

Fig. 2. Hierarchical synthesis of two-dimensional polymers via Ullmann-type reactions on $\mathrm{Au}(111)$. (a) Formation of rectangular porphyrin networks with chains as intermediate structures. Adapted with permission from Ref. ${ }^{47}$, copyright 2012, Nature Chemistry. (b) On-surface synthesis of porous phenylene networks using dimers as intermediate structures. Adapted with permission from Ref. ${ }^{35}$, copyright 2014, ACS. (c) Hierarchical synthesis of carbonyl-bridged triphenylamine networks using macrocycles and chains as intermediate structures. The STM image of the 2D triphenylamine based polymer with the corresponding DFT model is shown on the right. Adapted with permission from Ref. ${ }^{49}$, copyright 2017, Nature Communications.

A benefit of the hierarchical synthesis for the fabrication of well-ordered honeycomb networks was shown by Steiner et al., who used a more rigid precursor molecule and larger intermediate structures (see Fig. 2c). ${ }^{49}$ In the hierarchical synthesis, the diiodinated/brominated carbonyl-bridged triphenylamines (CTPA) formed covalently-linked six membered macrocycles and $1 \mathrm{D}$ chains as intermediate structures, which were subsequently connected to a porous 2D polymer as final product. Using brominated macrocycles and chains as reaction intermediates constrains the defects to the rim of the formed 2D networks, leaving the properties of the covalent polymer largely preserved. The covalent linkage of the well-ordered six membered 
macrocycles leads by design to the formation of honeycomb networks. The only possible defect would be a combination of 4-8 membered ring pairs, which were, however, not observed likely due to the large strain resulting from the inherent rigidity of the CTPA moieties. Pentagonal and heptagonal defects at the periphery of the porous graphene islands may be created by interlinking short chains before the second reaction occurs. Hence, covalent linking of macrocycles form defect-free 2D networks after the second reaction step, while chains are still susceptible to form pentagonal and heptagonal defects. Unfortunately, there is currently no method to tune the selectivity towards the macrocycle formation. The key of this hierarchical approach is that macrocycles can be fabricated via a bottom-up approach with high structural quality and rarely show other polygons than hexagons, whose geometry is commensurate with the symmetry of the molecule. The weak, non-covalent interactions among the macrocycles makes them structurally controllable and leads to equilibrated structures. This was also observed previously in the on-surface synthesis of hyperbenzene ${ }^{50}$ and honeycombene ${ }^{51}$ and recently for the diiodo-substituted triphenylamines $^{49}$ (see Fig. 3). In addition, the self-assembly of intermediate macrocycles and chains is essential to achieve structurally well-ordered covalent 2D networks, because undesired structural elements can segregate to the periphery of the network.

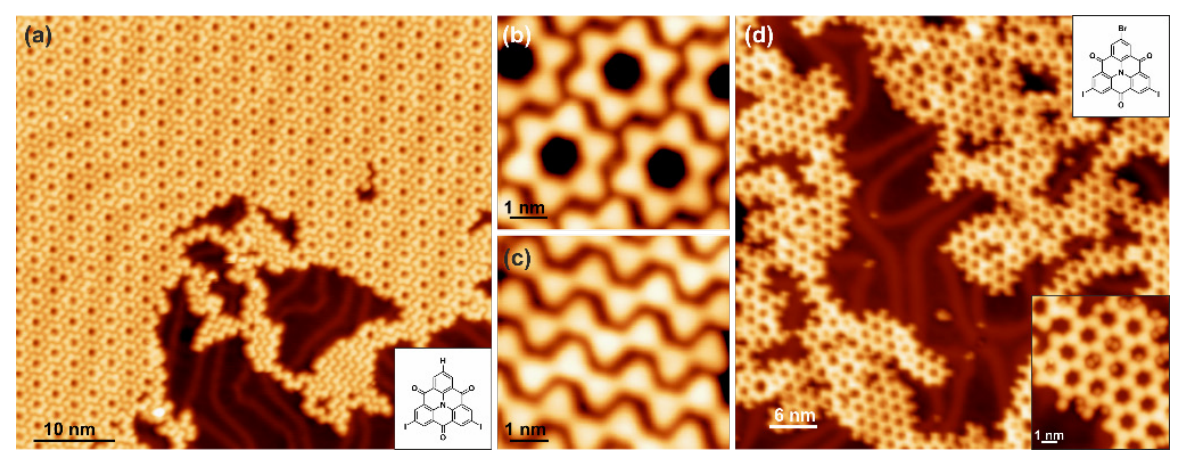

Fig. 3. (a-c) Self-assembly of macrocycles (b) and chains (c) fabricated by on-surface synthesis from diiodo-substituted carbonyl-bridged triphenylamine precursor molecules on $\mathrm{Au}(111)$. (d) $\mathrm{Hi}$ erarchically synthesized $2 \mathrm{D}$ polymer with carbonyl-functionalized pores on $\mathrm{Au}(111)$. Adapted with permission from Ref. ${ }^{49}$, copyright 2017, Nature Communications.

\section{Electronic structure of 2D polymers}

Much of the research of organic 2D polymers is driven by their unique electronic properties predicted by DFT. This includes the expectation to correlate the geometric structure with electronic properties. ${ }^{15}$ While the electronic properties of 1D structures fabricated by on-surface synthesis have thoroughly been characterized with several surface science techniques including scanning tunneling spectroscopy (STS) and ARPES, 5, 7-8, 52 similar approaches to study the band structure in two- 
dimensional $\pi$-conjugated systems remain largely unexplored. The concept of band gap engineering in $2 \mathrm{D}$ is especially interesting as the number of intermolecular connections scales linearly with the oligomer length in $1 \mathrm{D}$ but superlinearly in $2 \mathrm{D}$, which leads to a different HOMO-LUMO gap (HLG) for otherwise similar polymers (see Fig. 4) ${ }^{53}$ More specifically, the number of connections (conjugated links $k$ ) in 1D structures with the oligomer length $n$ is $k=n-1$ and in 2D structures $k=2 \sqrt{ } n(\sqrt{ } n-1)$.
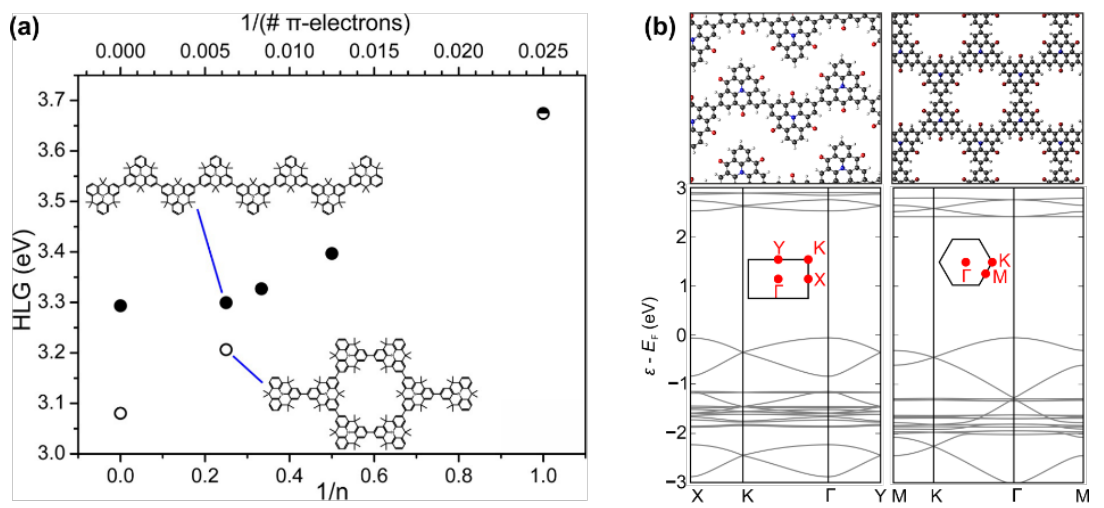

Fig. 4. (a) Evolution of the HOMO-LUMO gap (HLG) calculated by DFT (B3LYP) as a function of oligomer size for 1D and 2D dimethyl-bridged triphenylamine structures. Reprinted with permission from Ref. ${ }^{53}$, copyright 2017, ACS. (b) DFT band structures (HSE) for 1D (left) and 2D (right) carbonyl-bridged triphenylamine structures revealing a decrease of the band gap of about $120 \mathrm{meV}$ in the 2D case. Adapted with permission from Ref. ${ }^{49}$, copyright 2017, Nature Communications.

A number of DFT studies reported the dependence of the HOMO-LUMO gap on the $1 \mathrm{D}$ oligomer length and compared it also to the corresponding $2 \mathrm{D}$ structures. ${ }^{53-}$ ${ }^{56}$ Gutzler et al. ${ }^{53}$ systematically studied by DFT the HLG of polymer structures previously fabricated by on-surface synthesis experiments as a function of oligomer size and revealed the interplay between the dimensionality and the effective conjugation. One of the main conclusions of that DFT study was, that the HLG of 2D conjugated polymers is always smaller than that of the $1 \mathrm{D}$ counterpart, but the difference critically depends on the connectivity between the repeat units and varied between $0.1 \mathrm{eV}$ and $1 \mathrm{eV}$. The electron delocalization in most of the studied 2D polymers is limited by cross-conjugation (as in meta-substituted benzene) or by a large twist angle between the $\pi$-orbitals, although several of the reported $2 \mathrm{D}$ polymers possess a continuous network of $s p^{2}$-carbons. In contrast, only a few experimental surface science studies have addressed the changes in the electronic structure from the precursor molecule to oligomers and $2 \mathrm{D}$ networks on metals. ${ }^{55,}$ 57-58 Wiengarten et al. confirmed the reduction of the HLG in the transition from molec- 
ular precursors to 1D structures fabricated by surface-assisted dehydrogenative homocoupling of porphine molecules. ${ }^{57}$ Similarly, Cardenas et al. used a surface-confined Ullmann-type polymerization to fabricate 2D networks of polythiophene and showed in ultraviolet photoemission spectra (UPS) experiments that the HOMO of the molecular overlayer shifts towards the Fermi level by $0.6 \mathrm{eV}$ upon polymerization. ${ }^{55}$ However, the functional groups of the precursor molecules may play a decisive role in such experiments. Morchutt et al. ${ }^{58}$ showed in a combined STS/UPS study that in the polymerization reaction of 1,3,5-tris(4-carboxyphenyl)-benzene via decarboxylation on $\mathrm{Cu}(111)$ the carboxyl groups in the precursor molecule give rise to empty states that lie closer to the Fermi level than the empty states of polymerized structures. This is in contrast to the LUMO stabilization and shift toward the Fermi level as a result of the extending $\pi$-conjugation.

Structural control with atomic precision is prerequisite for studying the electronic structure of extended 2D polymer networks, because their band structure sensitively depends on the specific atomic configuration and size. The hierarchical approach presented by Steiner et al. ${ }^{49}$ yields $2 \mathrm{D}$ and $1 \mathrm{D}$ polymers built from identical building blocks with satisfactory structural quality (see Fig. 5). It provides therefore a direct comparison between the electronic structure of bottom-up fabricated 2D polymers and their analogous 1D counterparts, which has so far only been explored theoretically for extended $\pi$-systems ${ }^{53}$. STS experiments showed that the triphenylaminebased 2D networks are organic semiconductors with a bandgap of $2.45 \pm 0.09 \mathrm{eV}$, which is compared to the HOMO-LUMO gap of the monomer of $3.91 \pm 0.03 \mathrm{eV}$ significantly smaller. Further, it was concluded that the band gap in the CTPA compounds changes most from the monomer to the 1D chains, while it only decreases around $170 \mathrm{meV}$ from $1 \mathrm{D}$ to $2 \mathrm{D}$ structures. The decrease of the band gap from the monomer to the $2 \mathrm{D}$ polymer is a result of the increased effective $\pi$-conjugation length, which indicates an efficient conjugation among the triphenylamine building blocks within the nanostructure. The small difference between the band gaps of 1D and $2 \mathrm{D}$ compared to $0 \mathrm{D}$ and $1 \mathrm{D}$ shows that the $\pi$-electron delocalization length is only little increased between 1D and 2D CTPA polymers. In addition, constant height $\mathrm{d} I / \mathrm{d} V$ maps at the energies of the observed valence band edge (VBE) and conduction band $(\mathrm{CB})$ states provided further insights into the localization of the states. While the VBE shows an almost homogeneous electron density across the monomer backbone, the first unfilled states are localized near or at the newly formed $\mathrm{C}-\mathrm{C}$ bond.

The band gap of the triphenylamine-based networks obtained by DFT calculations at the HSE level are in good agreement with the experimentally measured values. ${ }^{49}$ In addition, band structure calculations provided further insights to the reduction of the band gap from 1D to 2D structures. In the 1D case, the band dispersion of the interacting $\pi$-bands around the Fermi level is decreased along $\overline{K Y}$ compared with the $2 \mathrm{D}$ case (Fig. $4 \mathrm{~b}$ ). This is observed in the valence band and (to a smaller extent) in the conduction band, leading to an overall decrease of the band gap by $0.12 \mathrm{eV}$. Moreover, the dispersionless band of the conduction band edge is missing in the $1 \mathrm{D}$ case. 

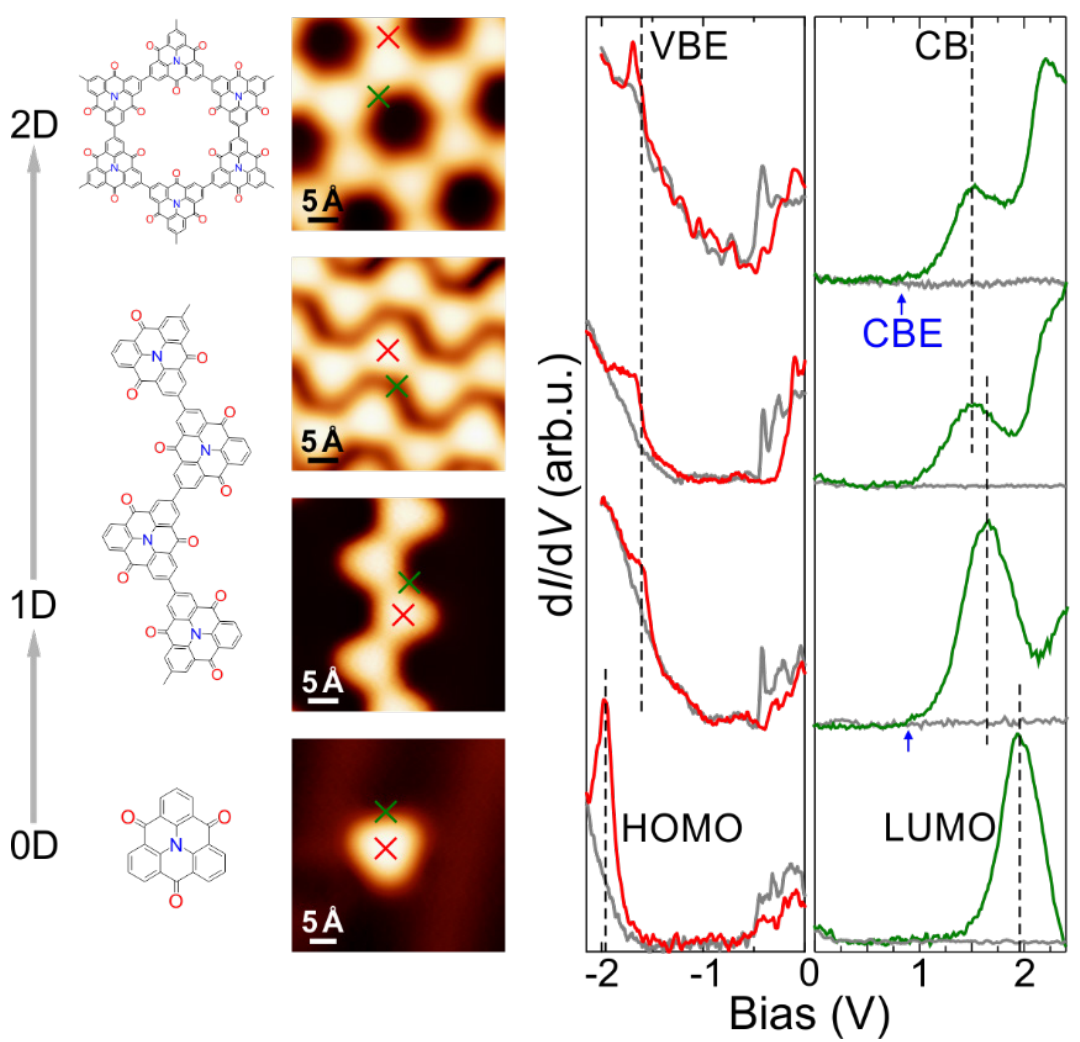

Fig. 5. $\mathrm{d} / / \mathrm{d} V$ curves measured on the porous $2 \mathrm{D}$ polymer, 1D chains, and CTPA monomer. The STM images indicate the location of the recorded STS spectra of the valence band edge (VBE, red) and the conduction band (CB, green) of the 2D network (top), self-assembled $1 \mathrm{D}$ chains ( $2^{\text {nd }}$ row), and isolated $1 \mathrm{D}$ chains ( $3^{\text {rd }}$ row), and the corresponding HOMO and LUMO of the CTPA monomer (bottom). The grey spectra represent the reference on the bare $\mathrm{Au}(111)$ surface. The blue arrows indicate the onset of the conduction band edge (CBE). Adapted with permission from Ref. ${ }^{49}$, copyright 2017, Nature Communications.

The inherent porous structure of 2D polymers leads by itself already to promising electronic properties. The pores induce a band gap that can be controlled for instance by the pore size, density, and geometry. ${ }^{14}$ In addition the electronic properties can be further tuned by either including dopant atoms in the carbon backbone ${ }^{59}$ or by trapping dopants in the pores via host-guest chemistry. Mostly carbon- and nitrogen-containing ${ }^{59-60} 2 \mathrm{D}$ porous polymers have been studied so far to avoid competing interactions among the functional groups during the reaction. With suitable precursors, there is no inherent limitation, however, to extend the surface-assisted synthesis to more complex 2D polymers that feature functionalized pores. ${ }^{49,61-62}$ The functional groups facing the pores can be used to selectively bind molecules through host-guest chemistry. Most of the host-guest chemistry experiments in 
physiosorbed networks on solid surfaces have focused to date on supramolecular networks. ${ }^{63}$ The reversible nature of the non-covalent interaction leads frequently to alterations in structure and pore sizes by the inclusion of guest molecules. ${ }^{64-65} \mathrm{In}$ contrast, the geometry and pore sizes of the covalently linked porous frameworks can hardly be impacted by the accommodation of guest species independent of their concentration and therefore provide more stability. Two-dimensional nanoporous covalent organic framework have been used to control the organization of various host molecules at the solid-liquid interface ${ }^{66-67}$ and in $\mathrm{UHV}^{68}$.

\section{Summary and Outlook}

On-surface polymerization is a successful method in the bottom-up fabrication of novel covalent nanostructures that are to some extent otherwise synthetically not accessible. While the structural control to obtain long-range ordered structures is commonly achieved in 1D structures, it is still challenging in 2D structures. One of the main reasons is the kinetically driven and irreversible formation of topological defects in form of various polygons, e.g. irregular pentagonal or heptagonal pores in hexagonal networks. Some preprogrammed synthesis strategies such as cyclotrimerization reactions or a hierarchical synthesis showed promise to suppress the defect formation, however so far with limited scalability. The long-range order is a strict requirement for any type of application, since crystallinity is necessary for the promotion of a defined band structure. Despite the many exciting electronic properties predicted by DFT, only a few experimental surface science studies have so far reported on the electronic structure of 2D polymers. Recently, the change in the HOMO-LUMO gap from the precursor molecule to oligomers has been shown for a few systems. STS experiments on hierarchically synthesized structures unveiled a narrowing of the band gap with increasing dimensionality. For applications of 2D polymers in devices, the $2 \mathrm{D}$ polymers need to be electrically decoupled. This requires the use of insulating surfaces either by in situ synthesis or subsequent transfer. First experiments on calcite surfaces have shown that on-surface synthesis on bulk insulator surfaces is feasible, however strategies to form well ordered 2D networks remain to be explored. ${ }^{69-70}$ In the other hand, the surface supported networks can be also decoupled via intercalation of halogens. ${ }^{71-73}$ In conclusion, the research on the surface synthesis of $2 \mathrm{D}$ polymer networks is a growing field, which is promising in particular for the synthesis of novel 2D carbon allotropes beyond graphene, such as graphyne or graphdiyne.

Acknowledgments The fruitful collaboration with Prof. Dr. Milan Kivala and Prof. Dr. Andreas Görling on the on-surface synthesis of 2D polymers at the Friedrich-Alexander University Erlangen-Nürnberg is gratefully acknowledged. Financial support by the German Research Foundation (DFG) through the Collaborative Research Center SFB 953 "Synthetic Carbon Allotropes" and 
the Cluster of Excellence EXC 315 "Engineering of Advanced Materials" at the Friedrich-Alexander University Erlangen-Nürnberg and the ERC Starting Grant SURFLINK (contract No. 637831) is acknowledged.

\section{References}

(1) Sakamoto, J.; van Heijst, J.; Lukin, O.; Schlüter, A. D. Two-Dimensional Polymers: Just a Dream of Synthetic Chemists? Angew. Chem. Int. Ed. 2009, 48, 1030-1069.

(2) Colson, J. W.; Dichtel, W. R. Rationally Synthesized Two-Dimensional Polymers. Nat. Chem. 2013, 5, 453-465.

(3) Cai, J.; Ruffieux, P.; Jaafar, R.; Bieri, M.; Braun, T.; Blankenburg, S.; Muoth, M.; Seitsonen, A. P.; Saleh, M.; Feng, X., et al. Atomically Precise Bottom-up Fabrication of Graphene Nanoribbons. Nature 2010, 466, 470-473.

(4) Talirz, L.; Ruffieux, P.; Fasel, R. On-Surface Synthesis of Atomically Precise Graphene Nanoribbons. Adv. Mater. 2016, 28, 6222-6231.

(5) Söde, H.; Talirz, L.; Gröning, O.; Pignedoli, C. A.; Berger, R.; Feng, X.; Müllen, K.; Fasel, R.; Ruffieux, P. Electronic Band Dispersion of Graphene Nanoribbons Via FourierTransformed Scanning Tunneling Spectroscopy. Phys. Rev. B 2015, 91, 045429.

(6) Cai, J.; Pignedoli, C. A.; Talirz, L.; Ruffieux, P.; Söde, H.; Liang, L.; Meunier, V.; Berger, R.; Li, R.; Feng, X., et al. Graphene Nanoribbon Heterojunctions. Nat. Nano. 2014, 9, 896900.

(7) Chen, Y.-C.; de Oteyza, D. G.; Pedramrazi, Z.; Chen, C.; Fischer, F. R.; Crommie, M. F. Tuning the Band Gap of Graphene Nanoribbons Synthesized from Molecular Precursors. ACS Nano 2013, 7, 6123-6128.

(8) Chen, Y. C.; Cao, T.; Chen, C.; Pedramrazi, Z.; Haberer, D.; de Oteyza, D. G.; Fischer, F. R.; Louie, S. G.; Crommie, M. F. Molecular Bandgap Engineering of Bottom-up Synthesized Graphene Nanoribbon Heterojunctions. Nat. Nano. 2015, 10, 156-160.

(9) Kawai, S.; Saito, S.; Osumi, S.; Yamaguchi, S.; Foster, A. S.; Spijker, P.; Meyer, E. Atomically Controlled Substitutional Boron-Doping of Graphene Nanoribbons. Nat. Commun. 2015, 6, 8098 .

(10) Cloke, R. R.; Marangoni, T.; Nguyen, G. D.; Joshi, T.; Rizzo, D. J.; Bronner, C.; Cao, T.; Louie, S. G.; Crommie, M. F.; Fischer, F. R. Site-Specific Substitutional Boron Doping of Semiconducting Armchair Graphene Nanoribbons. J. Am. Chem. Soc. 2015, 137, 88728875 .

(11) van der Lit, J.; Boneschanscher, M. P.; Vanmaekelbergh, D.; Ijäs, M.; Uppstu, A.; Ervasti, M.; Harju, A.; Liljeroth, P.; Swart, I. Suppression of Electron-Vibron Coupling in Graphene Nanoribbons Contacted Via a Single Atom. Nat. Commun. 2013, 4, 2023.

(12) Held, P. A.; Fuchs, H.; Studer, A. Covalent-Bond Formation Via on-Surface Chemistry. Chem. Eur. J. 2017, 23, 5874-5892.

(13) Whitelam, S.; Tamblyn, I.; Haxton, T. K.; Wieland, M. B.; Champness, N. R.; Garrahan, J. P.; Beton, P. H. Common Physical Framework Explains Phase Behavior and Dynamics of Atomic, Molecular, and Polymeric Network Formers. Phys. Rev. X 2014, 4, 011044.

(14) Pedersen, T. G.; Flindt, C.; Pedersen, J.; Mortensen, N. A.; Jauho, A.-P.; Pedersen, K. Graphene Antidot Lattices: Designed Defects and Spin Qubits. Phys. Rev. Lett. 2008, 100, 136804.

(15) Adjizian, J.-J.; Briddon, P.; Humbert, B.; Duvail, J.-L.; Wagner, P.; Adda, C.; Ewels, C. Dirac Cones in Two-Dimensional Conjugated Polymer Networks. Nat. Commun. 2014, 5 , 5842.

(16) Adjizian, J.-J.; Lherbier, A.; M.-M. Dubois, S.; Botello-Mendez, A. R.; Charlier, J.-C. The Electronic and Transport Properties of Two-Dimensional Conjugated Polymer Networks Including Disorder. Nanoscale 2016, 8, 1642-1651. 
(17) Malko, D.; Neiss, C.; Viñes, F.; Görling, A. Competition for Graphene: Graphynes with Direction-Dependent Dirac Cones. Phys. Rev. Lett. 2012, 108, 086804.

(18) Perepichka, D. F.; Rosei, F. Extending Polymer Conjugation into the Second Dimension. Science 2009, 323, 216.

(19) Blankenburg, S.; Bieri, M.; Fasel, R.; Müllen, K.; Pignedoli, C. A.; Passerone, D. Porous Graphene as an Atmospheric Nanofilter. Small 2010, 6, 2266-2271.

(20) Xiang, Z.; Cao, D.; Dai, L. Well-Defined Two Dimensional Covalent Organic Polymers: Rational Design, Controlled Syntheses, and Potential Applications. Polymer Chemistry 2015, 6, 1896-1911.

(21) Narita, A.; Wang, X.-Y.; Feng, X.; Mullen, K. New Advances in Nanographene Chemistry. Chem. Soc. Rev. 2015, 44, 6616-6643.

(22) Li, Y.; Xu, L.; Liu, H.; Li, Y. Graphdiyne and Graphyne: From Theoretical Predictions to Practical Construction. Chem. Soc. Rev. 2014, 43, 2572-2586.

(23) Gourdon, A. On-Surface Covalent Coupling in Ultrahigh Vacuum. Angew. Chem. Int. Ed. 2008, 47, 6950-6953.

(24) Fan, Q.; Gottfried, J. M.; Zhu, J. Surface-Catalyzed C-C Covalent Coupling Strategies toward the Synthesis of Low-Dimensional Carbon-Based Nanostructures. Acc. Chem. Res. 2015, 48, 2484-2494.

(25) Dong, L.; Liu, P. N.; Lin, N. Surface-Activated Coupling Reactions Confined on a Surface. Acc. Chem. Res. 2015, 48, 2765-2774.

(26) Björk, J.; Hanke, F. Towards Design Rules for Covalent Nanostructures on Metal Surfaces Chem. Eur. J. 2014, 20, 928-934.

(27) Lindner, R.; Rahe, P.; Kittelmann, M.; Gourdon, A.; Bechstein, R.; Kühnle, A. Substrate Templating Guides the Photoinduced Reaction of $\mathrm{C}_{60}$ on Calcite. Angew. Chem. Int. Ed. 2014, 53, 7952-7955.

(28) Palma, C.-A.; Diller, K.; Berger, R.; Welle, A.; Björk, J.; Cabellos, J. L.; Mowbray, D. J.; Papageorgiou, A. C.; Ivleva, N. P.; Matich, S., et al. Photoinduced C-C Reactions on Insulators toward Photolithography of Graphene Nanoarchitectures. J. Am. Chem. Soc. 2014, 136, 4651-4658.

(29) Kawai, S. Revealing Mechanical and Structural Properties of Molecules on Surface by HighResolution Atomic Force Microscopy. Polymer Journal 2017, 49, 3-11.

(30) Gross, L. Recent Advances in Submolecular Resolution with Scanning Probe Microscopy. Nat. Chem. 2011, 3, 273-278.

(31) Zwaneveld, N. A. A.; Pawlak, R.; Abel, M.; Catalin, D.; Gigmes, D.; Bertin, D.; Porte, L. Organized Formation of 2D Extended Covalent Organic Frameworks at Surfaces. J. Am. Chem. Soc. 2008, 130, 6678-6679.

(32) Côté, A. P.; Benin, A. I.; Ockwig, N. W.; O'Keeffe, M.; Matzger, A. J.; Yaghi, O. M. Porous, Crystalline, Covalent Organic Frameworks. Science 2005, 310, 1166-1170.

(33) Weigelt, S.; Busse, C.; Bombis, C.; Knudsen, M. M.; Gothelf, K. V.; Lægsgaard, E.; Besenbacher, F.; Linderoth, T. R. Surface Synthesis of 2D Branched Polymer Nanostructures. Angew. Chem. Int. Ed. 2008, 47, 4406-4410.

(34) Schlögl, S.; Heckl, W. M.; Lackinger, M. On-Surface Radical Addition of Triply Iodinated Monomers on $\mathrm{Au}(111)$ - the Influence of Monomer Size and Thermal Post-Processing. Surf. Sci. 2012, 606, 999-1004.

(35) Eichhorn, J.; Nieckarz, D.; Ochs, O.; Samanta, D.; Schmittel, M.; Szabelski, P. J.; Lackinger, M. On-Surface Ullmann Coupling: The Influence of Kinetic Reaction Parameters on the Morphology and Quality of Covalent Networks. ACS Nano 2014, 8, 7880-7889.

(36) Bieri, M.; Nguyen, M.-T.; Gröning, O.; Cai, J.; Treier, M.; Aït-Mansour, K.; Ruffieux, P.; Pignedoli, C. A.; Passerone, D.; Kastler, M., et al. Two-Dimensional Polymer Formation on Surfaces: Insight into the Roles of Precursor Mobility and Reactivity. J. Am. Chem. Soc. 2010, 132, 16669-16676.

(37) Grill, L.; Dyer, M.; Lafferentz, L.; Persson, M.; Peters, M. V.; Hecht, S. Nano-Architectures by Covalent Assembly of Molecular Building Blocks. Nat. Nano. 2007, 2, 687-691. 
(38) Bieri, M.; Treier, M.; Cai, J.; Ait-Mansour, K.; Ruffieux, P.; Groning, O.; Groning, P.; Kastler, M.; Rieger, R.; Feng, X., et al. Porous Graphenes: Two-Dimensional Polymer Synthesis with Atomic Precision. Chem. Comm. 2009, 6919-6921.

(39) Liu, J.; Ruffieux, P.; Feng, X.; Müllen, K.; Fasel, R. Cyclotrimerization of Arylalkynes on $\mathrm{Au}(111)$. Chem. Comm. 2014, 50, 11200-11203.

(40) Yang, B.; Björk, J.; Lin, H.; Zhang, X.; Zhang, H.; Li, Y.; Fan, J.; Li, Q.; Chi, L. Synthesis of Surface Covalent Organic Frameworks Via Dimerization and Cyclotrimerization of Acetyls. J. Am. Chem. Soc. 2015, 137, 4904-4907.

(41) Liu, J.; Ruffieux, P.; Feng, X.; Müllen, K.; Fasel, R. Cyclotrimerization of Arylalkynes on $\mathrm{Au}(111)$. Chem. Comm. 2014, 50, 11200-11203.

(42) Eichhorn, J.; Strunskus, T.; Rastgoo-Lahrood, A.; Samanta, D.; Schmittel, M.; Lackinger, M. On-Surface Ullmann Polymerization Via Intermediate Organometallic Networks on $\mathrm{Ag}(111)$. Chem. Comm. 2014, 50, 7680-7682.

(43) Biradha, K.; Santra, R. Crystal Engineering of Topochemical Solid State Reactions. Chem. Soc. Rev. 2013, 42, 950-967.

(44) Ruffieux, P.; Wang, S.; Yang, B.; Sánchez-Sánchez, C.; Liu, J.; Dienel, T.; Talirz, L.; Shinde, P.; Pignedoli, C. A.; Passerone, D., et al. On-Surface Synthesis of Graphene Nanoribbons with Zigzag Edge Topology. Nature 2016, 531, 489-492.

(45) Basagni, A.; Sedona, F.; Pignedoli, C. A.; Cattelan, M.; Nicolas, L.; Casarin, M.; Sambi, M. Molecules-Oligomers-Nanowires-Graphene Nanoribbons: A Bottom-up Stepwise onSurface Covalent Synthesis Preserving Long-Range Order. J. Am. Chem. Soc. 2015, 137, 1802-1808.

(46) Zhang, H.; Franke, J.-H.; Zhong, D.; Li, Y.; Timmer, A.; Arado, O. D.; Mönig, H.; Wang, H.; Chi, L.; Wang, Z., et al. Surface Supported Gold-Organic Hybrids: On-Surface Synthesis and Surface Directed Orientation. Small 2014, 10, 1361-1368.

(47) Lafferentz, L.; Eberhardt, V.; Dri, C.; Africh, C.; Comelli, G.; Esch, F.; Hecht, S.; Grill, L. Controlling on-Surface Polymerization by Hierarchical and Substrate-Directed Growth. Nat. Chem. 2012, 4, 215-220.

(48) Shi, K. J.; Zhang, X.; Shu, C. H.; Li, D. Y.; Wu, X. Y.; Liu, P. N. Ullmann Coupling Reaction of Aryl Chlorides on $\mathrm{Au}(111)$ Using Dosed $\mathrm{Cu}$ as a Catalyst and the Programmed Growth of 2D Covalent Organic Frameworks. Chem. Comm. 2016, 52, 8726-8729.

(49) Steiner, C.; Gebhardt, J.; Ammon, M.; Yang, Z.; Heidenreich, A.; Hammer, N.; Görling, A.; Kivala, M.; Maier, S. Hierarchical on-Surface Synthesis and Electronic Structure of Carbonyl-Functionalized One- and Two-Dimensional Covalent Nanoarchitectures. Nat. Commun. 2017, 8, 14765.

(50) Fan, Q.; Wang, C.; Han, Y.; Zhu, J.; Hieringer, W.; Kuttner, J.; Hilt, G.; Gottfried, J. M. Surface-Assisted Organic Synthesis of Hyperbenzene Nanotroughs. Angew. Chem. Int. Ed. 2013, 52, 4668-4672.

(51) Chen, M.; Shang, J.; Wang, Y.; Wu, K.; Kuttner, J.; Hilt, G.; Hieringer, W.; Gottfried, J. M. On-Surface Synthesis and Characterization of Honeycombene Oligophenylene Macrocycles. ACS Nano 2017, 11, 134-143.

(52) Vasseur, G.; Fagot-Revurat, Y.; Sicot, M.; Kierren, B.; Moreau, L.; Malterre, D.; Cardenas, L.; Galeotti, G.; Lipton-Duffin, J.; Rosei, F., et al. Quasi One-Dimensional Band Dispersion and Surface Metallization in Long-Range Ordered Polymeric Wires. Nat. Commun. 2016, 7, 10235 .

(53) Gutzler, R.; Perepichka, D. F. $\pi$-Electron Conjugation in Two Dimensions. J. Am. Chem. Soc. 2013, 135, 16585-16594.

(54) Wen, J.; Luo, D.; Cheng, L.; Zhao, K.; Ma, H. Electronic Structure Properties of TwoDimensional $\pi$-Conjugated Polymers. Macromolecules 2016, 49, 1305-1312.

(55) Cardenas, L.; Gutzler, R.; Lipton-Duffin, J.; Fu, C.; Brusso, J. L.; Dinca, L. E.; Vondracek, M.; Fagot-Revurat, Y.; Malterre, D.; Rosei, F., et al. Synthesis and Electronic Structure of a Two Dimensional $\pi$-Conjugated Polythiophene. Chem. Sci. 2013, 4, 3263-3268. 
(56) Baryshnikov, G. V.; Minaev, B. F.; Karaush, N. N.; Minaeva, V. A. The Art of the Possible: Computational Design of the 1D and 2D Materials Based on the Tetraoxa[8]Circulene Monomer. RSC Advances 2014, 4, 25843-25851.

(57) Wiengarten, A.; Seufert, K.; Auwärter, W.; Ecija, D.; Diller, K.; Allegretti, F.; Bischoff, F.; Fischer, S.; Duncan, D. A.; Papageorgiou, A. C., et al. Surface-Assisted Dehydrogenative Homocoupling of Porphine Molecules. J. Am. Chem. Soc. 2014, 136, 9346-9354.

(58) Morchutt, C.; Björk, J.; Straßer, C.; Starke, U.; Gutzler, R.; Kern, K. Interplay of Chemical and Electronic Structure on the Single-Molecule Level in 2D Polymerization. ACS Nano 2016, 10, 11511-11518.

(59) Sánchez-Sánchez, C.; Brüller, S.; Sachdev, H.; Müllen, K.; Krieg, M.; Bettinger, H. F.; Nicolaï, A.; Meunier, V.; Talirz, L.; Fasel, R., et al. On-Surface Synthesis of BN-Substituted Heteroaromatic Networks. ACS Nano 2015, 9, 9228-9235.

(60) Bieri, M.; Blankenburg, S.; Kivala, M.; Pignedoli, C. A.; Ruffieux, P.; Müllen, K.; Fasel, R. Surface-Supported 2D Heterotriangulene Polymers. Chem. Comm. 2011, 47, 10239-10241

(61) Faury, T.; Dumur, F.; Clair, S.; Abel, M.; Porte, L.; Gigmes, D. Side Functionalization of Diboronic Acid Precursors for Covalent Organic Frameworks. CrystEngComm 2013, 15, 2067-2075

(62) Xu, L.; Cao, L.; Guo, Z.; Zha, Z.; Lei, S. Side-Functionalized Two-Dimensional Polymers Synthesized Via on-Surface Schiff-Base Coupling. Chem. Comm. 2015, 51, 8664-8667.

(63) Teyssandier, J.; Feyter, S. D.; Mali, K. S. Host-Guest Chemistry in Two-Dimensional Supramolecular Networks. Chem. Comm. 2016, 52, 11465-11487.

(64) Kong, X.-H.; Deng, K.; Yang, Y.-L.; Zeng, Q.-D.; Wang, C. H-Bond Switching Mediated Multiple Flexibility in Supramolecular Host-Guest Architectures. J. Phys. Chem. C 2007, $111,17382-17387$

(65) Banerjee, K.; Kumar, A.; Canova, F. F.; Kezilebieke, S.; Foster, A. S.; Liljeroth, P. Flexible Self-Assembled Molecular Templates on Graphene. J. Phys. Chem. C 2016, 120, 87728780.

(66) Sun, J.; Zhou, X.; Lei, S. Host-Guest Architectures with a Surface Confined Imine Covalent Organic Framework as Two-Dimensional Host Networks. Chem. Comm. 2016, 52, 86918694.

(67) Cui, D.; MacLeod, J. M.; Ebrahimi, M.; Rosei, F. Selective Binding in Different Adsorption Sites of a 2D Covalent Organic Framework. CrystEngComm 2017.

(68) Blunt, M. O.; Russell, J. C.; Champness, N. R.; Beton, P. H. Templating Molecular Adsorption Using a Covalent Organic Framework. Chem. Comm. 2010, 46, 7157-7159.

(69) Kittelmann, M.; Nimmrich, M.; Lindner, R.; Gourdon, A.; Kühnle, A. Sequential and SiteSpecific on-Surface Synthesis on a Bulk Insulator. ACS Nano 2013, 7, 5614-5620.

(70) Lindner, R.; Kühnle, A. On-Surface Reactions. ChemPhysChem 2015, 16, 1582-1592.

(71) Rastgoo-Lahrood, A.; Björk, J.; Lischka, M.; Eichhorn, J.; Kloft, S.; Fritton, M.; Strunskus, T.; Samanta, D.; Schmittel, M.; Heckl, W. M., et al. Post-Synthetic Decoupling of onSurface-Synthesized Covalent Nanostructures from Ag(111). Angew. Chem. Int. Ed. 2016, $55,7650-7654$.

(72) Rastgoo-Lahrood, A.; Lischka, M.; Eichhorn, J.; Samanta, D.; Schmittel, M.; Heck1, W. M.; Lackinger, M. Reversible Intercalation of Iodine Monolayers between on-Surface Synthesised Covalent Polyphenylene Networks and $\mathrm{Au}(111)$. Nanoscale 2017, 9, 49955001

(73) Peyrot, D.; Silly, F. On-Surface Synthesis of Two-Dimensional Covalent Organic Structures Versus Halogen-Bonded Self-Assembly: Competing Formation of Organic Nanoarchitectures. ACS Nano 2016, 10, 5490-5498. 